it with the parameter which is already is been present and then it stores and evaluated on basis of that.

\section{Challenges in face Recognition}

\section{Technique}

1-The character must be at certain distance so that it can clearly capture and also itrequires that the character must not move for some time.

1-Also there are very few high recognition face databases available in the market so recognizing all is still a big task.

2-The major difference is that evaluating this is that there is a lot difference in the system along with the real life and that does not match enough.

\section{Working}

The face recognition technique consists of three basic steps they are:

1-Face detection- This technique consist of using the camera feature where the camera captures the real life images of the face and stores them in the data.

2-Face tracking- There are specific tools which are been used in this these tools consist of techniques where the data can be easily manipulated and also the data can be can be verified by the person or specialist

3-Face recognition-This is the best and the most important part in this technique because in this the faces which is been verified by the tracking process is been cleaned up along with that the image is been compared with lot more images in the database so that to contain a similar kind of image.

\section{Conclusion}

From this we can conclude that face recognition is a technique which has very much limitation as well as problem than too this is the emerging technique in security and technology purpose. But as the time is been going out this technique is becoming more and more advance and much more accurate than ever. Hopefully we have great techniques regarding face recognition technology and we can expect a better technique in the nearby future.

\section{Reference}

1. H. Yu, J. Yang, "A direct LDA algorithm for high-dimensional data - with application of face recognition", Pattern Recognition, vol. 34, no. 10, pp. 2067-2070, 2001.

2. W. S. Lee, H. J. Lee, J. H. Chung, "Wavelet-based FLD for face recognition", Circuits and Systems Proc. 43rd, vol. 2, pp. 734-737, 2000.

3. T. Morris, V. Chauhan, "Facial feature tracking for cursor control", Journal of Network and Computer Applications, vol. 29, no. 1, pp. 62-80, 2006.

4. J. Kim, K. R. Park, J. J. Lee, S. R. LeClair, "Intelligent process control via gaze detection technology", Engineering Applications of Artificial Intelligence, vol. 13, no. 5, pp. 577-587, 2000. 